\title{
Parasitic Rheumatism among Patients Infected with Intestinal Parasites
}

\author{
Eman M Hussein ${ }^{1 *}$, Eman M El-Hamshary ${ }^{1}$, Aziza S Omar² ${ }^{2}$ Amira B Mokhtar ${ }^{1}$ \\ ${ }^{1}$ Departments of Parasitology, Faculty of Medicine, Suez Canal University, Ismailia, Egypt \\ ${ }^{2}$ Rheumatology and Rehabilitation, Faculty of Medicine, Suez Canal University, Ismailia, Egypt \\ *Corresponding Author: Eman M Hussein, Departments of Parasitology, Faculty of Medicine, Suez Canal University, Ismailia, Egypt.
}

Received: June 24, 2019; Published: July 12, 2019

DOI: $10.31080 /$ ASGIS.2019.02.0059

\begin{abstract}
Introduction: Many intestinal parasites can induce a variety of rheumatic syndromes as a result of direct infiltration of musculoskeletal structures or an immune mediated mechanism.

Objectives: In this study, patients having unexplained rheumatic pain were examined to identifying the frequency of intestinal parasitic infection among them and which of them fulfilling parasitic rheumatism criteria.

Methodology: Stool samples of 107 patients having un explained rheumatic pain were examined to explore patients with parasitic rheumatism and rheumatologic blood investigation beside plan $\mathrm{X}$ ray for the affected joints were done.

Results: The results showed that 50 patients out of 107 had parasitic infection. Cryptosporidium infection was the commonest (48.0\%) followed by C. cayetanensis (32\%), G. lamblia (24\%), B. hominis (20\%) and E. histolytica (8\%). Beside Microsporidia, S. mansoni, A. lumbricoides and S. stercoralis were $4 \%$ for each. Some patients had mixed and triple parasitic infection. Infected parasitic patients fulfilling parasitic rheumatism criteria were 16/50 (32\%). Giardiasis patients having parasitic rheumatism constituted 8/16 (50\%) and were the commonest. The other eight (50\%) patients had Cryptosporidium, C. cayetanensis, E. histolytica/dispar and S. stercoralis. Two patients per each parasite. Giardiasis patients with parasitic rheumatism were females. Half of them $(50 \%)$ were < 15 years, complained of mono-articular joint pain, while the other were adults, complained of poly-articular joint pain.

Conclusion: Due to similarity of musculoskeletal presentations of parasitic diseases to many rheumatic diseases; consequently the importance of parasitic infection as an underlying cause of rheumatic syndromes especially in developing countries must be studied in patients undergoing evaluation for rheumatic complaints.
\end{abstract}

Keywords: Parasitic Rheumatism; Rheumatic Syndromes

\section{Introduction}

Rheumatic syndromes, including inflammatory arthritis, inflammatory myositis, and vasculitis have been described among multiple different parasitic infections of all parasitic divisions including protozoa, nematode, and platy-helminthes [1,2]. Rheumatic pain is the main clinical manifestation of rheumatic syndromes and is defined as; pain involving articular (related to joints) and/or extraarticular structures (related to tendons and muscles) [3]. Evidence for underlying parasitic infection should be sought in patients presenting with unexplained or atypical rheumatic syndromes $[4,5]$. Diagnosis of parasitic rheumatism is based on the demonstration of infection with a pathogenic parasite, lack of response to antiinflammatory agents, especially non steroidal anti-inflammatory drugs (NSAD), and improvement following anti-parasitic therapy with eradication of the parasite [1].

Many terms were introduced to describe the condition, e.g. reactive arthritis [6], rheumatic syndromes associated with parasites
[2], parasitic arthritis, parasitic rheumatism [4,7] where the causative pathogen is the parasite, followed after free interval with a characteristic pattern of arthropathy as a reaction to the presence of the parasite [8]. During that process, usually in 1 to 3 weeks, antigens are transported to the synovial tissue. This may occur intra-cellularly within mononuclear or other phagocytosing cells, as immune complexes, or even in the form of free antigen [9-11]. The consequence is a CD4+ cell mediated reaction, manifested as acute arthritis [12], immune complex mediated cytotoxicity may also participate [10]. For chronic antigen induced arthritis, a periodic or continuous supply of the antigen is required [13]. Arthritis induced by parasitic infection is very polymorphic; symptoms are monoarticular, oligoarticular, or polyarticular, involving small, medium, and/or large joints. They can mimic the clinical picture of different inflammatory rheumatic diseases [7]. Essential criteria for diagnosis of parasitic rheumatism were inflammatory arthropathy, residence in an area of endemic parasitosis, absence of radiological changes, identification of a pathogenic parasite, inefficacy of anti- 
rheumatic drugs and efficacy of specific anti-parasitic treatment as well as supplemental criteria which were inflammatory synovial fluid, elevated erythrocyte sedimentation rate and peripheral eosinophilia [4].

Many intestinal parasites can induce a variety of rheumatic syndromes as a result of infiltration of musculoskeletal structures by parasites or an immune mediated mechanism with circulating immune complexes and immunoglobulin deposits in synovium in some cases $[1,7]$. Protozoan-type infections were usually described with muscular and/or articular manifestations and directly correlated to the presence of the organism $[5,14]$. Nematodes are the second group of intestinal parasites commonly associated with rheumatic manifestations [4].

Given the continued and growing number of patients at risk for parasitic infections, being in endemic area, and due to similarity of musculoskeletal presentations of parasitic diseases to many rheumatic diseases The aim of this study was identifying the prevalence of parasitic rheumatism due to intestinal parasites among patients with unexplained rheumatic pain.

\section{Subjects and Methods}

This study was designed to estimate the prevalence of parasitic rheumatism among intestinal parasitic infected patients complaining of unexplained rheumatic pain attending rheumatology and rehabilitation outpatient clinic in Suez Canal University Hospital. Patients were above 3 years old, both sexes, residence in an area of endemic parasitosis and inefficacy of anti-rheumatic drugs. All patients were subjected to history taking covering full demographic data (age, sex, residence, any special habits of food handling, and socioeconomic state), present complaint including articular and extra-articular complaints (site and duration of pain), symptoms of intestinal parasitic infections (diarrhea, dysentery, abdominal discomfort, and dyspepsia) and past history of previous parasitic infections.

Patients with parasitic rheumatism were defined according to the criteria of parasitic rheumatism which include; essential criteria which are inflammatory arthropathy, residence in an area of endemic parasitosis, absence of radiological changes, identification of a pathogenic parasite, inefficacy of anti-rheumatic drugs and efficacy of specific anti-parasitic treatment as well as supplemental criteria which are inflammatory synovial fluid, elevated erythrocyte sedimentation rate (ESR), and peripheral eosinophilia [4]. Complete Blood Count (CBC), ESR C-Reactive protein (CRP), Rheumatoid factor (RF) and Anti-nuclear antibody (ANA) were performed. Plain X-ray for the affected joints was performed. Stool samples were collected and examined. One stool specimen was collected from each patient for three successive days. All of the stool analysis and cultures were performed according to Garcia [15]. Each stool sample was subjected to direct smear examination and iodine staining. Stool sample was fixed in Schaudinn's fixative and stained with trichrome stain on the day of collection or preserved in PVA for later staining. Part of the fresh stool sample was preserved in formalin $10 \%$ (1-3 parts). These were examined using low and high powers of the light microscopy. For diagnosis of Cryptosporidium Sp., C. cayetanensis, Isospora and Microsporidia the same samples were examined using modified Acid-Fast trichrome stain. Stool culture Harada Mori filter paper strip culture was used to diagnosis of S. stercoralis larvae and hookworms. Treatment by suitable anti-parasitic drugs was prescribed to infected patients, according to stool examination followed by stool re-examination 3 weeks after finishing treatment to be sure of parasite elimination and to detect cases with drug resistance.

Statistical analysis: In qualitative data, Chi square test was used to test difference for significance. P value ( 2 tailed) was set at $<0.05$ for significant results and $<0.01$ for highly significant results.

\section{Results}

In this study 50 intestinal parasitic infected patients having unexplained rheumatic pain were investigated to identify the prevalence of parasitic rheumatism. The intestinal parasites were Cryptosporidium in $48.0 \%$, C. cayetanensis in 32\%, G. lamblia in 24\%, B. hominis in $20 \%$ and E. histolytica in $8 \%$ patients. Beside, Microsporidia, S. mansoni, A. lumbricoides and S. stercoralis were $4 \%$ cases for each (Tab.1). Some patients had mixed and triple parasitic infection. Infected parasitic patients fulfilling parasitic rheumatism criteria were $32 \%$. Giardiasis patients having parasitic rheumatism constituted 50\% beside the other 50\% that had Cryptosporidium, C. cayetanensis, E. histolytica/dispar and S. stercoralis (2 cases for each). The majority of giardiasis patients $66.6 \%$ fulfilling the criteria of parasitic rheumatism while, the remaining $33.4 \%$ were not (did not response). The responding giardiasis patients were females, 50\% were young age, complained of mono-articular joint pain and $25 \%$ of them were GIT symptomatic, while the other $50 \%$ were adults, complained of poly-articular joint pain and $25 \%$ were GIT symptomatic (Figure 1 and Table 2). Elevated ESR and CRP were detected in $66.7 \%$. E. histolytica/dispar infected patients fulfilling parasitic rheumatism criteria were complaining of polyarticular joint pain. Both of cryptosporidiosis and cyclosporiasis patients fulfilling the criteria of parasitic rheumatism were $<15$ years males, having oligo-articular pain, GIT symptomic and single infection. S. stercoralis patients were female adults, living in rural areas, GIT symptomatic, complained of poly-articular joint pain, and having elevated ESR, CRP with esinophilia. The results proved that giardiasis was the common parasitic infection associated with unexplained rheumatic pain. 


\begin{tabular}{|l|c|c|c|c|c|c|}
\hline \multicolumn{2}{|c|}{$\begin{array}{c}\text { Intestinal parasitic } \\
\text { infected patients }\end{array}$} & $\begin{array}{c}\text { Non parasitic } \\
\text { rheumatism } \\
\mathbf{3 4 / 5 0}(\mathbf{6 8 \% )}\end{array}$ & \multicolumn{2}{c|}{$\begin{array}{c}\text { Parasitic } \\
\text { rheumatism } \\
(16 / 50)\end{array}$} \\
\hline Name of parasite & No & $\%$ & No. & $\%$ & No. & $\%$ \\
\hline Cryptosporidium & 24 & 48 & 22 & 44 & 2 & 4 \\
\hline C. cayetanensis & 16 & 32 & 14 & 28 & 2 & 4 \\
\hline G. lamblia & 12 & 24 & 4 & 8 & 8 & 16 \\
\hline B. hominis & 10 & 20 & 10 & 20 & 0 & 0 \\
\hline $\begin{array}{l}\text { E. histolytica/ } \\
\text { dispar }\end{array}$ & 4 & 8 & 2 & 4 & 2 & 4 \\
\hline Microsporidia & 2 & 4 & 2 & 4 & 0 & 0 \\
\hline S. mansoni & 2 & 4 & 2 & 4 & 0 & 0 \\
\hline S. stercoralis & 2 & 4 & 0 & 0 & 2 & 4 \\
\hline A. lumbricoides & 2 & 4 & 2 & 4 & 0 & 0 \\
\hline
\end{tabular}

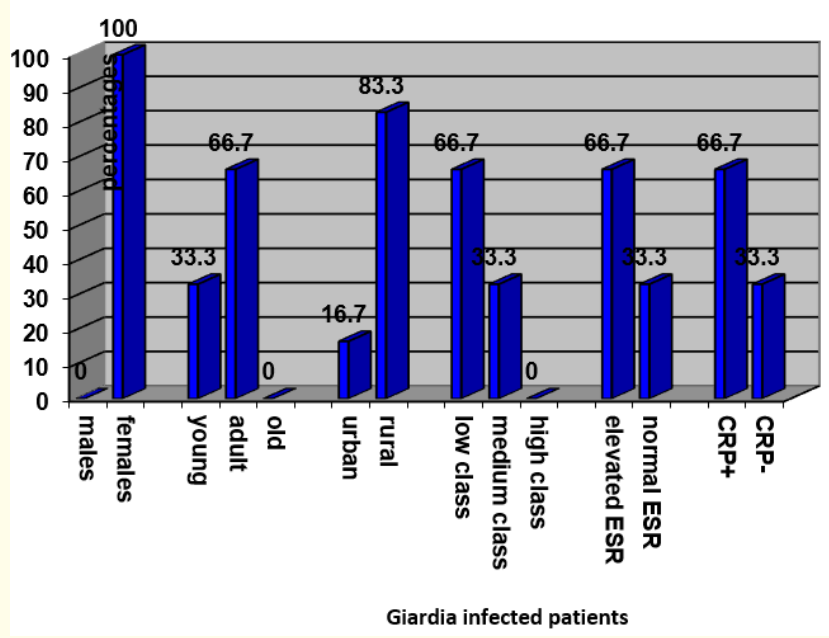

Table 1: Prevalence of different intestinal parasitic infection detected among patients with rheumatic pain and

Figure 1: The sex, ages, residence, socio-economic status and blood analysis of Giardia infected patients in the studied population. parasitic rheumatism.

\begin{tabular}{|c|c|c|c|c|c|c|c|c|c|}
\hline \multirow{2}{*}{\multicolumn{2}{|c|}{$\begin{array}{l}\text { Clinical characters of Giardiasis } \\
\text { patients }\end{array}$}} & \multicolumn{2}{|c|}{ Mono-articular } & \multicolumn{2}{|c|}{ Oligo-articular } & \multicolumn{2}{|c|}{ Poly-articular } & \multicolumn{2}{|c|}{ Total } \\
\hline & & \multirow{2}{*}{$\begin{array}{c}\text { No } \\
4\end{array}$} & \multirow{2}{*}{\begin{tabular}{|c|}
$\mathbf{\%}$ \\
33.3 \\
\end{tabular}} & \multirow{2}{*}{$\begin{array}{c}\text { No } \\
0 \\
\end{array}$} & \multirow{2}{*}{$\begin{array}{c}\% \\
0 \\
\end{array}$} & \multirow{2}{*}{$\begin{array}{c}\text { No } \\
4 \\
\end{array}$} & \multirow{2}{*}{$\begin{array}{c}\mathbf{\%} \\
33.3 \\
\end{array}$} & \multirow{2}{*}{$\begin{array}{c}\text { No } \\
8 \\
\end{array}$} & \multirow{2}{*}{$\begin{array}{c}\% \\
66.6\end{array}$} \\
\hline Anti-Parasitic & Responding & & & & & & & & \\
\hline & Non-Re. & 0 & 0 & 2 & 16.7 & 2 & 16.7 & 4 & 33.4 \\
\hline & Total & 4 & 33.3 & 2 & 16.7 & 6 & 50 & 12 & 100 \\
\hline \multirow[t]{3}{*}{ GIT } & Symptomatic & 2 & 16.7 & 0 & 0 & 2 & 16.7 & 4 & 33.4 \\
\hline & Asymptomatic & 2 & 16.6 & 2 & 16.7 & 4 & 33.3 & 8 & 66.6 \\
\hline & Total & 4 & 33.3 & 2 & 16.7 & 6 & 50 & 12 & 100 \\
\hline \multirow[t]{3}{*}{ Infection } & Single & 4 & 33.3 & 0 & 0 & 4 & 33.3 & 8 & 66.6 \\
\hline & Mixed & 0 & 0 & 2 & 16.7 & 2 & 16.7 & 4 & 33.4 \\
\hline & Total & 4 & 33.3 & 2 & 16.7 & 6 & 50 & 12 & 100 \\
\hline
\end{tabular}

Table 2: The relation between responding to anti-parasitic treatment, GIT symptoms, type of infection in Giardia infected patients and joint affection pattern.

\section{Discussion}

In the present study $32 \%$ patients gave good response with complete relief of rheumatic complaints and disappearance of the parasite from their stool. While, in $68 \%$ patients the rheumatic complaint persisted despite full course treatment of the parasitic infection (Table 1). This data goes in agreement with, Di Pietro [13] he reported that muscular and/or articular manifestations are not unusual in protozoal type infections. Also, a well-defined rheumatic syndrome occurs in a variety of parasitic diseases with distinct reported rates were recorded $[5,16,17]$. Controversy, some authors considered this condition of parasitic rheumatism to be rare $[7,18]$. The suspicion that parasitic antigens are sometimes responsible for arthritis in some susceptible individuals was supported by the rapid resolution of symptoms after anti-parasitic therapy.

In the present study, the prevalence of parasitic rheumatism among giardiasis patients was $66.7 \%$. In agreement with these data, Giardia was reported as a cause of reactive arthritis by Goobar [19] who described musculoskeletal manifestations (acute polyarthiritis) of giardiasis in a series of 66 infected children aged 2-15 years with a prevalence rate of $36 \%$. Also, arthritis and arthralgia have been reported among a series of 10 children, GIT symptomatic, 70\% of them were males with cysts of $G$. lamblia in feces with elevation on ESR was found in 60\%, while CRP was negative [20]. However, an acute poly-arthiritis in adult female patient associated with giardiasis [21]. In contrast giardiasis is consider a rare cases of arthritis [22].

In the present study, only 2 cryptosporidiosis patients having parasitic rheumatism were found (Table). Many reports confirmed that cryptosporidiosis was a common cause of reactive arthritis especially in children [18,23-26]. Meanwhile, only four cases of Cryptosporidium reactive arthritis in adults were reported worldwide, two were HIV patients one had mono-articular joint pain [27], the 
last one had oligo-articular joint affection [28]. Elevated ESR and CRP were found in $16.7 \%$ and $8.3 \%$ respectively of Cryptosporidium infected patients in this study (Figure). In the study of Lee., et al. [29] all cases infected with Cryptosporidium had elevated ESR and some of them had elevated CRP. Also, ESR and CRP of cryptosporidiosis reactive arthritis patients were elevated in several reports $[25,28]$. This elevation in ESR and CRP was considered to reflect some types of inflammation existing inside the body [29].

In the present study, only two cyclosporiasis patients were fulfilling the criteria of parasitic rheumatism. In agreement with our results two studied found a relation between $C$. cayetanensis infection and musculoskeletal manifestations such as Guillian Barrie syndromes and Reiter's syndromes [30,31]. These syndromes were mainly secondary conditions to cyclosporiasis or sometimes constitutional symptoms such as muscles aches or joints aches [5,32].

In the present study, $50 \%$ of the patients ( 2 cases) with E. histolytica/dispar infection gave good response to anti-parasitic treatment. The difference was statistically non significant. These data was supported with a studies that demonstrated that infection with $E$. histolytica has been associated with autoimmune phenomena and development of symmetrical poly-arthritis very similar to rheumatoid and reactive arthritis [4,5]. In some cases, organisms have been observed in synovial fluid, suggesting invasive infection $[4,33]$.

As regards to helminthes, in the present work, S. stercoralis was present in 2 cases $(1.9 \%)$. In agreement with these results many reports involving patients with either oligo or poly-arthritis in association with intestinal Strongyloides infection were presented [34]. It usually involves the lower extremity large joints but sometimes the sacroiliac or upper extremity large joints as well [6,3538]. Although Strongyloides larvae have been observed in a synovial biopsy specimen, suggesting an infectious type of arthritis most authors consider this to be a reactive arthritis [34,39,40]. All of the reported cases were recorded in developed countries in which the prevalence of intestinal parasites especially in immunocompetent patients is low. Although arthritis is regarded to be uncommon $[34,41]$. In a Japanese study on 299 patients, arthralgia was the most common symptom reported by the strongyloidiasis patients [42]. In case of developing countries, the prevalence of parasitic rheumatism could be higher where safety food and water supply may be absent. These findings may be explaining the high prevalence that was reported in this study.

In conclusion, given the continued and growing number of patients at risk for parasitic infections, being in endemic area, and due to similarity of musculoskeletal presentations of parasitic diseases to many rheumatic diseases; consequently the importance of parasitic infection as an underlying cause of rheumatic syndromes must be studied in patients undergoing evaluation for rheumatic complaints.

\section{Bibliography}

1. Bocanegra TS and Vasey FB. "Musculoskeletal syndromes in parasitic diseases". Rheumatic Diseases Clinics of North America 19.2 (1993): 505-513.

2. McGill PE. "Rheumatic syndromes associated with parasites". Baillière's Clinical Rheumatology 1 (1995): 201-213.

3. Kelley WN., et al. In: Kelley Textbook of Rheumatology 94th ed (2012).

4. Peng SL. "Rheumatic manifestations of parasitic diseases". Seminars in Arthritis and Rheumatism 31.14 (2002): 228-247.

5. Aikawa NE., et al. "Intestinal microsporidiosis: a hidden risk in rheumatic disease patients undergoing anti-tumor necrosis factor therapy combined with disease-modifying anti-rheumatic drugs?". Clinics 66.7 (2011): 1171-1175.

6. Bocanegra TS., et al. "Reactive arthritis induced by parasitic infestation". Annals of Internal Medicine 94.2 (1981): 207-209.

7. Doury P. "Is there a role for parasites in the etiology of inflammatory rheumatism?". Bulletin de l'Académie Nationale De Médecine 174.6 (1990): 743-751.

8. El-Araby S. "Arthropathies associated with parasitic infestations". Curr. Reco. Rheumatol. 1st edn 1 (2003): 40.

9. Salmi M., et al. "Dual binding capacity of mucosal immune blasts to mucosal and synovial endothelium in humans: dissection of the molecular mechanisms". Journal of Experimental Medicine 181. 1 (1995): 137-149.

10. Lahesmaa-Rantala R., et al. "Yersinia specific immune complexes in the synovial fluid of patients with yersinia triggered reactive arthritis". Annals of the Rheumatic Diseases 46.7 (1987): 510-514.

11. Lehtonen L., et al. "Synovial fluid muramic acid in acute inflammatory arthritis". British Journal of Rheumatology 33.12 (12994): 1127-1130.

12. Burmester GR., et al. "Immunology of reactive arthritides". Annual Review of Immunology 13 (1995) 229-250.

13. Toivanen P and Toivanen A. "Two Forms of Reactive Arthritis". Annals of the Rheumatic Diseases 58.12 (1999): 737-741.

14. Di Pietro A., et al. "Parasitic arthritis: a case report". Pediatria Medica e Chirurgica 18.2 (1996): 211-222. 
15. Garcia LS. "Diagnostic Medical Parasitology". 5th ed. Washington DC: ASM Press; (2007): 60-105.

16. McGill PE. "Geographically specific infections and arthritis, including rheumatic syndromes associated with certain fungi and parasites, Brucella species and Mycobacterium leprae". Best Practice and Research: Clinical Rheumatology 17.2 (2003): 289-307.

17. Crum--Cianflone NF. "Infection and musculoskeletal conditions: Infectious myositis". Best Practice and Research: Clinical Rheumatology 20.6 (2006): 1083-1097.

18. Sing A., et al. "Reactive arthritis associated with prolonged cryptosporidial infection". Journal of Infection 47 (2003): 181184.

19. Goobar, JP. “Joint symptoms in giardiasis". Lancet 7. 1 (1977): 1010-1011.

20. Meza-Ortíz FA. " Giardiasis-associated arthralgia in children”. 32.3 (2001): 248-250.

21. Shaw RA and Stevens MB. "The reactive arthritis of giardiasis. A case report”. JAMA 20.258 (1987): 2734-2735.

22. Krol A. "Giardia lamblia as a rare cause of reactive arthritis". Ugeskr Laeger. 175.49 (2013): 513.

23. Shepherd RC., et al. "Reactive arthritis complicating cryptosporidial infection". Archives of Disease in Childhood 64.5 (1989): 743-744.

24. Cron RQ and Sherry DD. "Reiter's syndrome associated with cryptosporidial gastroenteritis". Journal of Rheumatology 22.10 (1995): 1962-1963.

25. Rees JR., et al. "Persistent diarrhea, arthritis, and other complications of enteric infections: a pilot survey based on California Food Net surveillance, 1998-1999". Clinical Infectious Diseases 38 (2004): S311-317.

26. Hay EM., et al. "Reactive arthritis associated with Cryptosporidium enteritis”. The BMJ (Clin Res Ed). 295 (1987): 248.

27. Ozgül A., et al. "Sacroiliitis associated with Cryptosporidium parvum in an HLA-B27-negative patient". Rheumatology (OXford). 38.3 (1999): 288-289.

28. Collings S and Highton J. "Cryptosporidium reactive arthritis". NZMJ 117. 1200 (2004): 1023.

29. Lee JK., et al. "Prevalence of diarrhea caused by Cryptosporidium parvum in non-HIV patients in Jeollanam-do, Korea". The Korean Journal of Parasitology 43.3 (2005): 111-114.
30. Richardson RF., et al. "Guillain-Barré syndrome after Cyclospora infection”. Muscle Nerve 21.5 (1998): 669-671.

31. Connor BA., et al. "Reiter syndrome following protracted symptoms of Cyclospora infection”. Emerging Infectious Diseases 7.3 (2001): 453-454.

32. Ortega YR and Sanchez R. "Update on Cyclospora cayetanensis, a Food-Borne and Waterborne Parasite". Clinical Microbiology Reviews 23 (2010): 218-223.

33. Burnstein SL and Liakos S. "Parasitic rheumatism presenting as rheumatoid arthritis". Journal of Rheumatology 10.3 (1983): 514-515.

34. van Kuijk AW. "Early-onset polyarthritis as presenting feature of intestinal infection with Strongyloides stercoralis". Rheumatology 42.11 (2003): 1419-1420.

35. Patey 0., et al. "Arthritis associated with Strongyloides stercoralis". Scandinavian Journal of Infectious Diseases 22.2 (1990): 233-236.

36. Brocq 0., et al. "Reactive arthritis induced by Strongyloides stercoralis". Revue du rhumatisme 63.3 (1996): 217-219.

37. Masseau A., et al. "Strongyloides stercoralis infection simulating polyarteritis nodosa". Revue de Médecine Interne 26. 8 (2005): 661-663.

38. Richter J., et al. "Arthritis associated with Strongyloides stercoralis infection in a HLA B-27-positive African patients". Parasitology Research 99 (2006): 706-710.

39. Akoğlu T., et al. "Parasitic arthritis induced by Strongyloides stercoralis". Annals of the Rheumatic Diseases 43.3 (1984): 523-525.

40. Gullo A., et al. "A Strongyloides stercoralis infection presenting as arthritis of sternoclavicular joint". Modern Rheumatology 26.6 (2016): 981-983.

41. Ghotekar LH., et al. "Reactive arthritis, psoriasiform lesions and protein loosing enteropathy secondary to strongyloidiasis". The Journal of the Association of Physicians of India 51 (2003): 395-396.

42. Zaha O., et al. "Clinical study on symptoms in patients with strongyloidiasis”. Kansenshogaku Zasshi 66.10 (1992): 13781382.

Volume 2 Issue 6 August 2019

(C) All rights are reserved by Eman M Hussein., et al. 